\title{
Conservative treatment, plate fixation, or prosthesis for proximal humeral fracture. A prospective randomized study
}

Antti P Launonen ${ }^{1 *}$, Vesa Lepola ${ }^{1}$, Tapio Flinkkilä2 ${ }^{2}$ Niko Strandberg ${ }^{3}$, Johanna Ojanperä $^{4}$, Pekka Rissanen ${ }^{5}$, Antti Malmivaara ${ }^{6}$, Ville M Mattila ${ }^{1}$, Petra Elo ${ }^{7}$, Timo Viljakka ${ }^{8}$ and Minna Laitinen ${ }^{1}$

\begin{abstract}
Background: Proximal humerus fracture is the third most common fracture type after hip and distal radius fracture in elderly patients. A comprehensive study by Palvanen et al. demonstrated an increase in the annual fracture rate of $13.7 \%$ per year over the past 33 years. Should this trend continue, the fracture rate would triple over the next three decades. The increasing incidence of low-energy fractures raises questions about the optimal treatment in terms of functional outcome, pain, and rehabilitation time, as well as the economical impact. Despite the high incidence and costs of proximal humerus fractures, there is currently no valid scientific evidence for the best treatment method. Several publications, including a Cochrane review outline the need for high-quality, well-designed randomized controlled trials.
\end{abstract}

Methods/Design: The study is a prospective, randomized, national multi-center trial. The hypothesis of the trial is that surgical treatment of displaced proximal humerus fractures achieves better functional outcome, pain relief, and patient satisfaction compared to conservative treatment. The trial is designed to compare conservative and surgical treatment of proximal humerus fractures in patients 60 years and older. The trial includes two strata. Stratum I compares surgical treatment with locking plates to conservative treatment for two-part fractures. Stratum II compares multi-fragmented fractures, including three- and four-part fractures. The aim of Stratum II is to compare conservative treatment, surgical treatment with the Philos locking plate, and hemiarthroplasty with an Epoca prosthesis. The primary outcome measure will be the Disabilities of the Arm, Shoulder and Hand (DASH) score and the secondary outcome measures will be the EuroQol-5D (EQ-5D) value, OSS, Constant-Murley Score, VAS, and 15D. Recruiting time will be 3 years. The results will be analyzed after the 2-year follow-up period.

Discussion: This publication presents a prospective, randomized, national multi-center trial. It gives details of patient flow, randomization, aftercare and also ways of analysis of the material and ways to present and publish the results.

Trial registration: ClinicalTrials.gov identifier: NCT01246167

Keywords: Proximal, Humerus, Fracture, Conservative, Operative, Locking plate, Prosthesis, Philos, Epoca, RCT

\footnotetext{
* Correspondence: antti.launonen@pshp.fi

'Department of Orthopaedics, Tampere University Hospital, Teiskontie 35,

PL2000, Tampere 33521, Finland

Full list of author information is available at the end of the article
}

\section{Biomed Central}

(c) 2012 Launonen et al.; licensee BioMed Central Ltd. This is an Open Access article distributed under the terms of the Creative Commons Attribution License (http://creativecommons.org/licenses/by/2.0), which permits unrestricted use, distribution, and reproduction in any medium, provided the original work is properly cited. 


\section{Background}

Proximal humerus fracture is the third most common fracture type after hip and distal radius fracture in elderly patients [1-3]. Proximal humerus fracture accounts for approximately $4 \%$ of all fractures [1-3]. Approximately $85 \%$ of the patients are treated conservatively and will regain shoulder function without surgery [4]. Most of these fractures are stable and minimally or non-displaced osteoporotic fractures and they commonly occur in women [4]. The mechanism of low-energy injury in elderly patients is usually falling from standing height. In Finland in 2002, the age-adjusted fracture incidence in persons 60 years and older was 105 per 100,000 person-years [5]. A comprehensive study by Palvanen et al. demonstrated an increase in the annual fracture rate of $13.7 \%$ per year over the past 33 years [5]. Should this trend continue, the fracture rate would triple over the next three decades.

The increasing incidence of low-energy fractures raises questions about the optimal treatment in terms of functional outcome, pain, and rehabilitation time, as well as the economical impact. Despite the high incidence and costs of proximal humerus fractures, there is currently no valid scientific evidence for the best treatment method. Several publications, including a Cochrane review outline the need for high-quality, well-designed randomized controlled trials. The challenge for the future is to determine which patients will benefit from surgery and to establish surgical techniques that produce optimal results for each fracture type. The aim of this randomized controlled trial is to evaluate whether the outcome in patients over 60-years of age with displaced two-, three-, and four-part fractures of the proximal humerus is improved by surgical intervention.

\section{Diagnosis and treatment}

Diagnosis of proximal humerus fracture is based on clinical and radiologic findings and the mechanism of injury. A standard set of three radiographs from different views is generally obtained. The Neers's or AO (Arbeitsgemeinschaft für Osteosynthesefragen) classification systems are widely used to define these complex fractures [6]. Although these systems have been used extensively for many decades, their reliability has been challenged. The AO system categorizes the fracture types into 27 fracture patterns, making its use labor- and time-intensive and complicated (Müller 1990). The Codman-Hertel binary fracture description system does not address the fracture pathomechanism (Hertel 2004). The Codman-Hertel system was improved by Resch by adding the pathomechanism of the fracture to the classification [7]. In all classification systems, however, the intra- and inter-observer agreement are graded as poor or, at best, moderate [8]. Due to poor intra- and inter-observer agreement of the Neer's or AO classification systems, various radiographic protocols have been introduced to improve the diagnostic reliability of the classification. CT is often performed to facilitate treatment decisions.

Approximately $15 \%$ of patients with proximal humerus fracture are treated surgically [9]. Several fixation methods have been introduced, including Kirschner-wire fixation, screw fixation, plate fixation, intramedullary fixation, and prosthesis [10]. Currently, the locking plate system is the most frequently used method for fixation in two- and three-part fractures and a locking plate or prostheses is often used in displaced three- and four-part fractures in elderly patients [11]. With locking plates, the normal anatomy may be restored and the range of motion (ROM) is reported to recover up to $80 \%$ to $85 \%$ that of the healthy side $[10,12]$. The disadvantage of the locking plates includes a rather high complication rate of up to $49 \%$ [13]. A stable and usually pain-free shoulder is achieved with a prosthesis, but recovery of ROM is poor [10,12,14-17].

\section{Evaluation of treatment}

Tools that are widely used for measuring the mobility and usability of the shoulder include the Constant-Murley score; Disabilities of the Arm, Shoulder and Hand (DASH) questionnaire; Oxford Shoulder Score (OSS); and Visual Analog Scale (VAS). In addition, the EuroQol-5D (EQ-5D) and $15 \mathrm{D}$ questionnaires survey the patient's general quality of life through different questions pertaining to various areas of life and are widely used in medical trials [18]. The outcomes are indexed and are comparable with reference populations as well as with the patient's own results in other stages of the treatment. Finnish versions of the EQ5D and 15D have been validated $[19,20]$.

\section{Previous studies}

Although the literature on proximal humerus fractures is extensive, the majority of studies lack randomization, comparators, and independent evaluation, which makes it impossible to draw clinically meaningful conclusions [21]. Recent publications include three well done randomized, controlled trials. Olerud et al. carried out randomized controlled trials on three-part fractures, comparing nonsurgical treatment with angle-stable plates in elderly patients. The results indicated advantages in functional outcome and health-related quality of life favoring the locking plate, but the clinical significance remains unclear [22]. Fjalestad et al. studied displaced three and four-part fractures in patients over 60 years of age. They found no evidence that surgical treatment with an angle-stable device provided better results than conservative treatment [23]. Others have reported conflicting results. Olerud et al. studied displaced four-part fractures in elderly patients. They compared hemiarthroplasty and conservative treatment and found that arthroplasty provided a significant advantage in terms of quality of life. The main advantage was less pain although there was no difference in ROM [24]. 
In 2009, Hanson et al. published functional results of 160 patients treated conservatively. After a 12-month follow-up, the difference compared to the healthy side was 8.2 points measured with the Constant-Murley score. The difference in the DASH score was 10.2 points, which is below the minimal detectable change. Non-union risk was $7.0 \%$, with smokers having a 5.5 times greater risk than nonsmokers [25].

The updated Cochrane meta-analysis published in 2010 was unable to provide guidelines for treating proximal humerus fractures due to a lack of solid evidence. Three- and four-part fractures in patients over 60 years of age are especially challenging as scientific consensus on their treatment has yet to be established [21].

\section{Methods/Design}

The present randomized controlled trial is designed to compare conservative and surgical treatment of proximal humerus fractures. The trial includes two strata. Stratum I compares surgical treatment with locking plates to conservative treatment for two-part fractures. Stratum II compares multi-fragmented fractures, including three- and four-part fractures. The aim of Stratum II is to compare conservative treatment, surgical treatment with the Philos locking plate $\left(\right.$ Synthes $\left.^{\circledR}\right)$, and hemiarthroplasty with an Epoca prosthesis (Synthes ${ }^{\circledR}$ ).

\section{Hypothesis}

The study is a prospective, randomized, national multicenter trial. The hypothesis of the trial is that surgical treatment of displaced proximal humerus fractures achieves better functional outcome, pain relief, and patient satisfaction compared to conservative treatment in terms of ROM, and Constant-Murley, DASH, OSS, EQ-5D, 15D, and VAS scores. Subgroup analysis will be performed in an effort to obtain limit values for specific treatments of different age and fracture groups. In addition, we hypothesize that shoulder function will improve for up to 1 year from the time of fracture, and after 1 year significant improvement will be arrested [26]. The primary outcome measure will be the Disabilities of the Arm, Shoulder and Hand (DASH) score and the secondary outcome measures will be the EuroQol-5D (EQ-5D) value, OSS, Constant-Murley Score, VAS, and 15D.

\section{Objectives}

The results of both strata will be analyzed and the results will be reported separately, as recommended by the CONSORT statement.

\section{Patients and methods Inclusion criteria}

- Low energy proximal humerus displaced (displacement more than $1 \mathrm{~cm}$ or 45 degrees) two-part fracture in which the fracture line emerges through the surgical (or anatomic) neck

- Low energy proximal humerus displaced (displacement more than $1 \mathrm{~cm}$ or 45 degrees) three- or four-part fracture

\section{Exclusion criteria}

- Refusal to participate in the study

- Under 60 years of age

- Not independent

- Dementia and/or institutionalized

- Does not understand written and spoken guidance in either Finnish or Swedish

- Pathologic fracture or a previous fracture of the same proximal humerus

- Alcoholism or drug addiction, e.g., in the emergency department, breathalyzer indicates blood alcohol concentration of more than $2 \%$

- Other injury to the same upper limb requiring surgery

- Major nerve injury (e.g., complete radial- or axillary nerve palsy)

- Rotator cuff tear arthropathy

- Open fracture

- Multi-trauma or -fractured patient

- Fracture dislocation or head-splitting fracture

- Non-displaced fracture

- Isolated fracture of the major or minor tubercle

- Gross displacement of the fracture fragments (no bony contact between fracture parts or the humerus shaft is in contact with the articular surface)

- Any medical condition that excludes surgical treatment

Patients with an x-ray verified proximal humerus fracture meeting the inclusion criteria will undergo a CT scan to assess the fracture classification. The scan area will include the entire scapula with the upper third of the humerus. Coronal, sagittal, and 3-dimensional volume reformats will be reconstructed. If the fracture meets the radiologic inclusion criteria, the patient will be invited to participate in the study. The patient will be informed of the study and will receive a written information sheet. When patients decide to participate in the study, they will be asked to fill-out a written informed consent form. The patients can withdraw from the study at any stage, on any grounds, and this will have no influence on the medical care given to the patients. If the patient is excluded from the study, information about age, sex, fracture type, reason for exclusion, medical condition, basic medication, and chosen treatment will be communicated to the research group using a case report form.

During hospitalization, the patient will be asked to fill out, with help if necessary, the EQ-5D, 15D, DASH, and OSS, and basic patient questionnaires with a VAS to determine their baseline characteristics. The patient's medical 
history, medication, and surgery will be recorded by a research nurse or researcher on a medical case report form. Potential primary complications will be recorded (e.g., nerve injury).

\section{Randomization}

All patients will be randomized by a Tampere University Hospital research coordinator who will not attend the study. Patients with a two-part fracture will be randomized to either conservative or plate-fixation groups. Patients with multi-fragmented fractures will be randomized to conservative, plate fixation, or prosthesis groups. Both fracture types will be randomized using a random number matrix in block allocation fashion. The blocks will be age-dependent because, based on the literature, age and functional outcome are associated [27]. The treatment allocations from the matrix will be sealed in an envelope. After the patient's enrollment in the study has been confirmed, the research physician will contact the research coordinator, who will open the envelope and the randomized treatment will be carried out. The research coordinator will monitor the study flow. An independent monitoring committee has not been established.

\section{Surgical technique}

The surgical procedures (plate fixation or hemiarthroplasty) are performed by shoulder-oriented orthopedic surgeons of the Tampere, Kuopio, Turku, and Oulu University hospitals. In this trial, we will use the Philos locking plate system (Synthes ${ }^{\circledR}$, Solothurn, Switzerland) and an uncemented Epoca fracture prosthesis (Synthes ${ }^{\circledR}$, Solothurn, Switzerland) with a hydroxyapatite coating. Additional cement fixation will be used if necessary. All patients randomized to surgical treatment will undergo the surgery within 2 weeks after the fracture. During the operation, the Neer's classification and evidence of potential rotator cuff rupture will be recorded.

Patients will be placed in the beach-chair position. Plexus anesthesia will be used when possible. The deltopectoral, deltoid-split, or minimally invasive plate osteosynthesis approach will be used. Cuff tendons will be routinely inspected and sutures passed through each tendon to ease the handling of the fragments. For plating, the fragments will be preliminarily reduced with sutures and k-wires. The plate will be placed lateral from the biceps groove and 5 $\mathrm{mm}$ distal from the tip of the tuberculum majus. Six to eight locking screws will be placed in the head and three conventional or locking screws will be placed in the shaft.

The prosthesis height will be determined from the medial rim of the articular fragment. Anatomic retroversion will be determined from the shaft configuration. Reaming will be performed until the trial stem is stable. If needed, a minimal amount of cement will be used to stabilize the stem. Head size is determined from the articular fragment. The offset will be left to neutral. Before tightening the cables underneath the tuberculi, bone grafts from the head fragment will be placed against the stem. Sutures will be knotted to secure the cuff.

Drainage will be left in if necessary and the wound closed in layers. The shoulder will be immobilized with a collarcuff in the operating theater.

\section{Conservative treatment}

Patients randomized to non-operative treatment will be instructed with regard to joint mobilization by a physiotherapist during hospitalization. Patients will receive a written aftercare protocol with detailed pictures. A collar-cuff or a sling will be used for 3 weeks to relieve pain. During the first 3 weeks, pendulum exercises are allowed, and free joint mobilization and normal limb activation throughout treatment will be strongly supported. Active ROM exercises, as allowed by pain, will begin at 3 weeks. Physiotherapist contacts will be arranged to begin 3 and 6 weeks after surgery and all patients will have 5 physiotherapist contacts within the 3 first months.

\section{Postoperative aftercare}

Patients operated with a plate will follow the same protocol as in conservative treatment. Patients treated with a prosthesis will wear a sling for 6 weeks. Two weeks postoperatively, they will begin pendulum movements. Free, active mobilization will be allowed at 6 weeks. Patients will be advised to mobilize their free joints from the beginning of the treatment and normal limb activation during aftercare will be supported. Contact with a hospital physiotherapist will begin after 3 and 6 weeks postoperatively and all patients will have 5 physiotherapist contacts within 3 months from the beginning of treatment. Patients will receive a detailed written aftercare protocol with instructional pictures and formal physiotherapy will be instructed before leaving the hospital.

\section{Follow-up}

Follow-up will be carried out at the orthopedic outpatient clinic of the hospital where the patient was primarily treated. The patients will visit the outpatient clinic at 6 weeks and 3 months. Ultrasound examination of the fractured shoulder to assess possible rotator cuff injury will be performed at the 3-month visit by an experienced musculoskeletal radiologist.

Orthopedic outpatient clinic visits will be continued if necessary. In addition to these visits, in each center a blinded physiotherapist or sports physiologist will perform a research examination at 6 months, and 1, 2, 5, and 10 years from the beginning of the treatment. Patients are supposed to wear a shirt to blind the examiner. During these visits, radiographs will be taken of the treated shoulder, ROM and ConstantMurley scores of both shoulders will be obtained, and the 
patient will complete the EQ-5D, 15D, DASH, and OSS questionnaires.

Should any adverse event demanding separate outpatient or inpatient care or surgery occur during the follow-up, an adverse event form will be completed within 24 hours of the execution of treatment. The information will be sent to the research coordinator. If the patient is not willing to continue in the study, or does not appear at appointments, or dies, a research discontinuation form will be completed. The study flow is outlined in Figure 1 and assessments and procedures are outlined in Table 1.

\section{Power analysis}

In Stratum I, when assuming an effect size of a 10-point difference in the DASH score and a standard deviation of 15 points, the estimated sample size is 37 patients (delta $=10$, $\mathrm{sd}=15$, alpha $=0.05$, power $=0.8$ ). Thus Stratum I requires 74 patients (2 comparison groups). In Stratum II, when assuming an effect size of a 10-point difference in the DASH score and a standard deviation of 15 points, the estimated sample size is 66 patients (ANOVA, alpha $=0.05$, power $=0.8$ ). The number of comparison groups is three. The total number of patients in Stratum II is 198. Thus, the total number of patients in the study will be 272 . We will assume a $10 \%$ drop-out rate in both groups; therefore, the total patient number required will be $299(81+218)$. In cases in which the patient changes to a different treatment group, they will be analyzed according to the intention-to-treat principle. SD value has been estimated after Gummerson et al. (2003). The article describes the minimal detectable change in

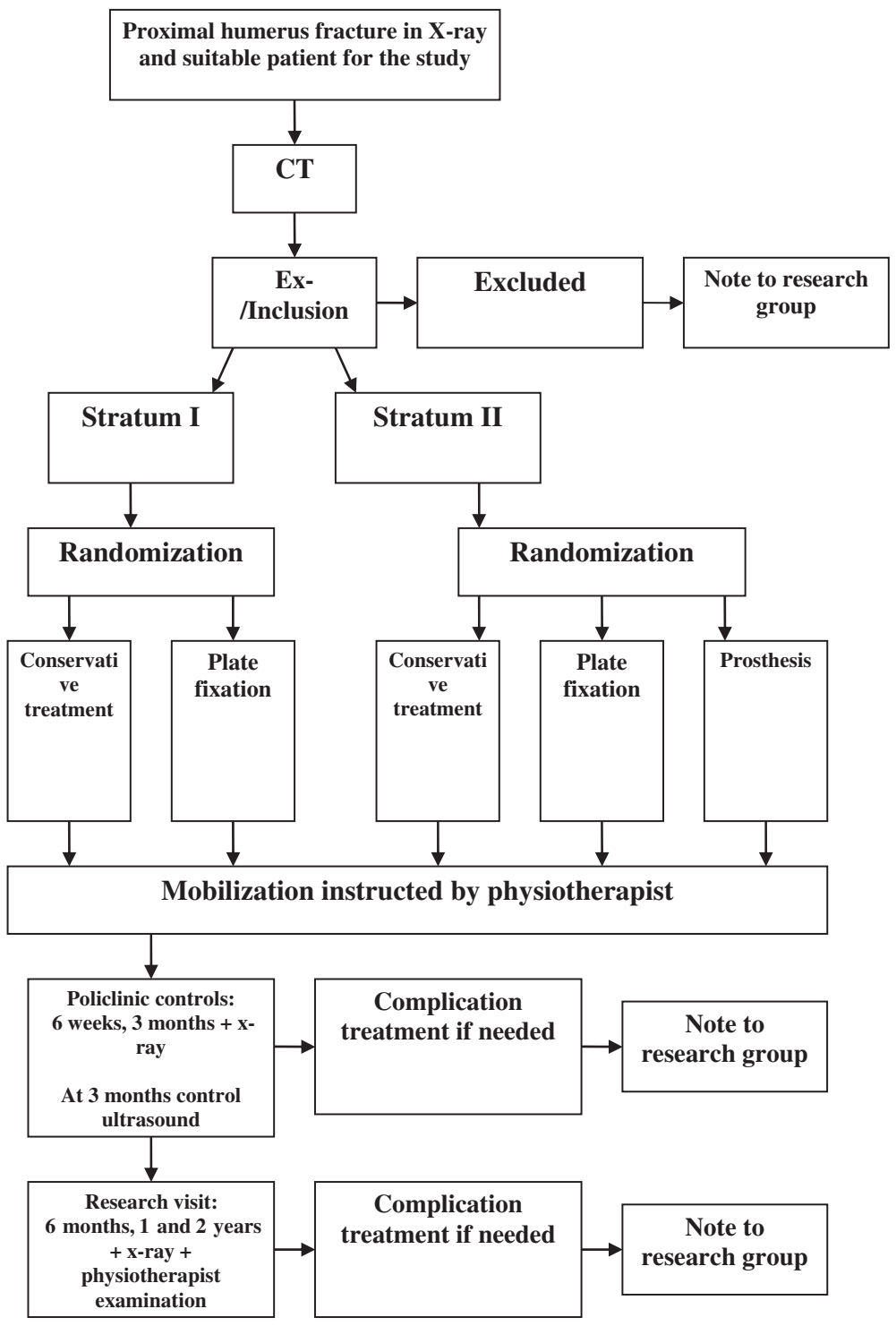

Figure 1 Study flow. Stratum I for 2 part fractures, Stratum II for 3 and 4 part fractures. 
Table 1 Assessments and procedures of the trial

\begin{tabular}{|c|c|c|c|c|c|c|}
\hline Assessment & Preoperative & 1. visit 6 weeks & 2. visit 3 months & 3. visit 6 months & 4. visit 1 year & 5.visit 2 years \\
\hline x-ray & $x$ & $x$ & $x$ & $x$ & $x$ & $x$ \\
\hline CT & $x$ & & & & & \\
\hline Ultrasound & & & $x$ & & & \\
\hline Ex-/inclusion & $x$ & & & & & \\
\hline Medical history & $x$ & & & & & \\
\hline Consent & $x$ & & & & & \\
\hline Questionnaire & $x$ & & $x$ & $x$ & $x$ & $x$ \\
\hline VAS-pain & $x$ & & & $x$ & $x$ & $x$ \\
\hline EQ-5D & $x$ & & & $x$ & $x$ & $x$ \\
\hline $15 D$ & $x$ & & & $x$ & $x$ & $x$ \\
\hline OSS & $x$ & & & $x$ & $x$ & $x$ \\
\hline DASH & $x$ & & & $x$ & $x$ & $x$ \\
\hline Constant-Murley Score & & & & $x$ & $x$ & $x$ \\
\hline Doctors visit & & $x$ & $x$ & & & \\
\hline Research visit & & & & $x$ & $x$ & $x$ \\
\hline
\end{tabular}

DASH as 10 points with an SD of 13 with a mean score of 15 points [28].

\section{Statistical analysis}

Differences between groups in continuous skewed main outcome variables will be analyzed by the Mann-Whitney U-test and t-test when variables are unskewed. Results are presented with $95 \%$ confidence intervals. Two-way-tables with the chi-square test will be used for dichotomous variables. Multivariate analysis will be conducted with regression analysis. In subgroup analysis the effect of age, sex, fracture group, smoking, and other diseases will be evaluated against the ROM, OSS, Constant-Murley, and overall quality of life after fracture.

\section{Analysis of the material}

All radiographs and CT scans will be sent to the research center at Tampere University Hospital.

All information gathered will be stored in a study registry at Tampere University Hospital. The registry is protected with passwords, and will be deleted 2 years after the end of the study.

\section{Ethics}

The trial protocol has been approved by the Ethics Committee of Pirkanmaa District Hospital. The study protocol and additional papers, including the consent form, patient information sheet, questionnaires, and case report form, have also been approved by the Ethics Committee (Approval number R10127). Permission to collect registry data and to combine it with the hospitalization data maintained by the NIHW will be requested from the NIHW and Social Insurance Institution.

\section{Time schedule}

Recruiting time will be 3 years. The results will be analyzed after the 2-year follow-up period. The final report will be published by the end of the year 2017 .

\section{Discussion}

This publication presents a prospctive, randomized, national multi-center trial. It gives details of patient flow, randomization, aftercare and also ways of analysis of the material and ways to present and publish the results.

\section{Competing interests}

The authors declare that they have no competing interests.

Authors' contributions

$A L, M L, T F, J O, N S$ and $T V$ are responsible for developing the trial. $A L, V M, T F$, $P E, P R, A M, N S, T V, V L$ and $M L$ drafted the protocol. AM will monitor and advise the methodologic aspects of the trial. PE is responsible for the radiologic aspects and $\mathrm{PR}$ is responsible for the economical aspect of the trial. VM performed the power calculations and determination of sufficient study group size and will perform the statistical analyses. All authors read and approved the final manuscript.

\section{Authors' information}

$\mathrm{ML}$ is the Principal Investigator of the study and she, as well as the CoPrincipal Investigators in Oulu and Turku - TF and NS, are experienced in carrying out randomized controlled trials. TF, VL and NS are also nationally respected shoulder surgeons. JO is Co-Principal Investigator at Kuopio University Hospital. AL is the main researcher and is responsible for coordinating the medical aspects and the practical side of the study. TV is a nationally respected senior shoulder surgeon and will aid in planning the study and instructing junior colleagues. PE is an experienced musculoskeletal radiologist and will provide advice on radiologic issues and is the head of the radiologic board of the trial. PR is Professor of Public Health of University of Tampere and is responsible for the economical aspects of the study. VM is Associate Professor in the Department of Traumatology. AM is a researcher at the National Institute for Health and Welfare. 


\section{Acknowledgements}

The research group thanks postdoctoral fellow Janne Pitkäniemi, Helsinki University, for his assistance with the statistical power analyses. In the end research group wants to give most sincere thanks for Dr Tore Fjalestad, Oslo University Hospital for his clear and recreational comments during the writing process.

\section{Funding}

The study is funded from each hospital's EVO funds for office, research and personnel expenses. EVO is a national government based non-commercial funding system. Funding from the Academy of Finland is requested.

\section{Author details}

'Department of Orthopaedics, Tampere University Hospital, Teiskontie 35, PL2000, Tampere 33521, Finland. 'Oulu University Hospital, Kajaanintie 50, PL21, Oulu 90029, Finland. ' ${ }^{3}$ Department of Orthopaedics and Traumatology, Turku University Hospital, Kiinamyllynkatu 4-8, PL 52, Turku 20521, Finland. ${ }^{4}$ Department of Traumatology and Hand Surgery, Kuopio University Hospital, Puijonlaaksontie 2, PL1777, Kuopio 70211, Finland. ${ }^{5}$ University of Tampere, Tampere School of Public Health, Tampere 33014, Finland. ${ }^{6}$ National Institute for Health and Welfare, Mannerheimintie 166, PL 30, 00271, Helsinki, Finland. ${ }^{7}$ Tampere University Hospital; Imaging Center, Biokatu 8, PL2000, Tampere 33521, Finland. ${ }^{8}$ Department of Hand Surgery, Tampere University Hospital, Teiskontie 35, PL2000, Tampere 33521, Finland.

\section{Received: 22 February 2012 Accepted: 31 August 2012}

Published: 7 September 2012

\section{References}

1. Lauritzen JB, Schwarz P, Lund B, McNair P, Transbol I: Changing incidence and residual lifetime risk of common osteoporosis-related fractures. Osteoporos Int 1993, 3(3):127-132.

2. Seeley DG, Browner WS, Nevitt MC, Genant HK, Scott JC, Cummings SR: Which fractures are associated with low appendicular bone mass in elderly women? The Study of Osteoporotic Fractures Research Group. Ann Intern Med 1991, 115(11):837-842.

3. Court-Brown CM, Caesar B: Epidemiology of adult fractures: A review. Injury 2006, 37(8):691-697.

4. Kristiansen B, Barfod G, Bredesen J, Erin-Madsen J, Grum B, Horsnaes MW, Aalberg JR: Epidemiology of proximal humeral fractures. Acta Orthop Scand 1987, 58(1):75-77.

5. Palvanen M, Kannus $P$, Niemi S, Parkkari J: Update in the epidemiology of proximal humeral fractures. Clin Orthop Relat Res 2006, 442:87-92.

6. Neer CS 2nd: Displaced proximal humeral fractures. I. Classification and evaluation. J Bone Joint Surg Am 1970, 52(6):1077-1089.

7. Resch H: [Fractures of the humeral head]. Unfallchirurg 2003, 106(8): 602-617.

8. Majed A, Macleod I, Bull AM, Zyto K, Resch H, Hertel R, Reilly P, Emery RJ: Proximal humeral fracture classification systems revisited. J Shoulder Elbow Surg 2011, 20(7):1125-1132.

9. Neer CS 2nd: Displaced proximal humeral fractures. II. Treatment of three-part and four-part displacement. J Bone Joint Surg Am 1970, 52 (6):1090-1103.

10. Lanting B, MacDermid J, Drosdowech D, Faber KJ: Proximal humeral fractures: a systematic review of treatment modalities. J Shoulder Elbow Surg 2008, 17(1):42-54

11. Murray IR, Amin AK, White TO, Robinson CM: Proximal humeral fractures: current concepts in classification, treatment and outcomes. J Bone Joint Surg Br 2011, 93(1):1-11.

12. Thanasas C, Kontakis G, Angoules A, Limb D, Giannoudis P: Treatment of proximal humerus fractures with locking plates: a systematic review. J Shoulder Elbow Surg 2009, 18(6):837-844.

13. Sproul RC, lyengar JJ, Devcic Z, Feeley BT: A systematic review of locking plate fixation of proximal humerus fractures. Injury 2011, 42(4):408-413.

14. Sudkamp N, Bayer J, Hepp P, Voigt C, Oestern H, Kaab M, Luo C, Plecko M, Wendt K, Kostler W, et al: Open reduction and internal fixation of proximal humeral fractures with use of the locking proximal humerus plate. Results of a prospective, multicenter, observational study. J Bone Joint Surg Am 2009, 91(6):1320-1328.
15. Solberg BD, Moon CN, Franco DP, Paiement GD: Surgical treatment of three and four-part proximal humeral fractures. J Bone Joint Surg Am 2009, 91(7):1689-1697.

16. Bastian JD, Hertel R: Osteosynthesis and hemiarthroplasty of fractures of the proximal humerus: outcomes in a consecutive case series. J Shoulder Elbow Surg 2009, 18(2):216-219.

17. Antuna SA, Sperling JW, Cofield RH: Shoulder hemiarthroplasty for acute fractures of the proximal humerus: a minimum five-year follow-up. J Shoulder Elbow Surg 2008, 17(2):202-209.

18. Vainiola T, Pettila V, Roine RP, Rasanen P, Rissanen AM, Sintonen $H$ : Comparison of two utility instruments, the EQ-5D and the 15D, in the critical care setting. Intensive Care Med 2010, 36(12):2090-2093.

19. Ohinmaa $\mathrm{A}$, Sintonen $\mathrm{H}$ : Inconsistencies and modelling of the Finnish EuroQol (EQ-5D) preference values. In EuroQol Plenary Meeting, 1-2 October 1998 Discussion papers Centre for Health Economics and Health Systems Research, University of Hannover, Germany. Edited by Greiner W, Grafvd Schulenburg J-M, Piercy J.: Uni-Verlag Witte; 1999:67-76.

20. Sintonen $\mathrm{H}$, Pekurinen $\mathrm{M}$ : Uses of $15 \mathrm{D}$-measure of health-related quality of life. In Health systems - the challenge of change. Proceedings of the 5th International Conference on System Science in Health Care. Prague June 29-July 3, 1992. Edited by Chytil MK, Duru G, van Eimeren W, Flagle CD. Prague: Omnipress; 1992:1071-1074.

21. Handoll HH, Ollivere BJ: Interventions for treating proximal humeral fractures in adults. Cochrane Database Syst Rev 2010, 12:CD000434.

22. Olerud $P$, Ahrengart $L$, Ponzer S, Saving J, Tidermark J: Internal fixation versus nonoperative treatment of displaced 3-part proximal humeral fractures in elderly patients: a randomized controlled trial. J Shoulder Elbow Surg 2011, 20(5):747-755.

23. Fjalestad T, Hole MO, Hovden IA, Blucher J, Stromsoe K: Surgical Treatment With an Angular Stable Plate for Complex Displaced Proximal Humeral Fractures in Elderly Patients: A Randomized Controlled Tria. J Orthop Trauma 2012, 26(2):98-106.

24. Olerud P, Ahrengart L, Ponzer S, Saving J, Tidermark J: Hemiarthroplasty versus nonoperative treatment of displaced 4-part proximal humeral fractures in elderly patients: a randomized controlled trial. J Shoulder Elbow Surg 2011, 20(7):1025-1033

25. Hanson B, Neidenbach $P$, de Boer P, Stengel D: Functional outcomes after nonoperative management of fractures of the proximal humerus. J Shoulder Elbow Surg 2009, 18(4):612-621.

26. Hodgson SA, Mawson SJ, Saxton JM, Stanley D: Rehabilitation of two-part fractures of the neck of the humerus (two-year follow-up). J Shoulder Elbow Surg 2007, 16(2):143-145.

27. Court-Brown CM, McQueen MM: Two-part fractures and fracture dislocations. Hand Clin 2007, 23(4)):397-414

28. Gummesson C, Atroshi I, Ekdahl C: The disabilities of the arm, shoulder and hand (DASH) outcome questionnaire: longitudinal construct validity and measuring self-rated health change after surgery. BMC Musculoskelet Disord 2003, 4:11.

doi:10.1186/1471-2474-13-167

Cite this article as: Launonen et al:: Conservative treatment, plate fixation, or prosthesis for proximal humeral fracture. A prospective randomized study. BMC Musculoskeletal Disorders 2012 13:167.

\section{Submit your next manuscript to BioMed Central and take full advantage of:}

- Convenient online submission

- Thorough peer review

- No space constraints or color figure charges

- Immediate publication on acceptance

- Inclusion in PubMed, CAS, Scopus and Google Scholar

- Research which is freely available for redistribution 